\title{
Construction and evaluation of nitric oxide generating vascular graft material loaded with organoselenium catalyst via layer-by-layer self-assembly
}

\author{
AN Jun ${ }^{1 \dagger}$, CHEN SiYuan $^{1 \dagger}$, GAO JingChen ${ }^{1}$, ZHANG Xu ${ }^{1}$, WANG YuanYuan ${ }^{1}$, LI YanDong ${ }^{2}$, \\ MIKHALOVSKY Sergey ${ }^{3}$, KONG DeLing ${ }^{1} \&$ WANG ShuFang ${ }^{1 *}$ \\ ${ }^{1}$ State Key Laboratory of Medicinal Chemical Biology, Key Laboratory of Bioactive Materials for Ministry of Education, College of Life Sci- \\ ences, Nankai University, Tianjin 300071, China; \\ ${ }^{2}$ Institute of Polymer Chemistry, College of Chemistry, Nankai University, Tianjin 300071 China; \\ ${ }^{3}$ School of Pharmacy and Biomolecular Sciences, University of Brighton, Brighton BN2 4GJ, UK
}

Received November 10, 2014; accepted March 12, 2015; published online May 25, 2015

\begin{abstract}
A new biomimetic material for artificial blood vessel with in situ catalytic generation of nitric oxide (NO) was prepared in this study. Organoselenium immobilized polyethyleneimine as NO donor catalyst and sodium alginate were alternately loaded onto the surface of electrospun polycaprolactone matrix via electrostatic layer-by-layer self-assembly. This material revealed significant NO generation when contacting NO donor S-nitrosoglutathione (GSNO). Adhesion and spreading of smooth muscle cells were inhibited on this material in the presence of GSNO, while proliferation of endothelial cells was promoted. In vitro platelet adhesion and arteriovenous shunt experiments demonstrated good antithrombotic properties of this material, with inhibited platelet activation and aggregation, and prevention of acute thrombosis. This study may provide a new method of improving cellular function and antithrombotic property of vascular grafts.
\end{abstract}

nitric oxide, catalytic generation, electrospinning, vascular graft, biocompatibility

Citation: $\quad$ An J, Chen SY, Gao JC, Zhang X, Wang YY, Li YD, Mikhalovsky S, Kong DL, Wang SF. Construction and evaluation of nitric oxide generating vascular graft material loaded with organoselenium catalyst via layer-by-layer self-assembly. Sci China Life Sci, 2015, 58: 765-772, doi: $10.1007 / \mathrm{s} 11427-015-4870-\mathrm{z}$

Cardiovascular diseases as coronary artery occlusion are now a serious threat to human health worldwide [1]. One of the effective treatments of these diseases is vascular graft implantation, and an inner surface with antithrombotic properties and rapid endothelialization is the key to success.

As a potent vasodilator and antiplatelet agent [2-4], nitric oxide (NO) has unique functions [5]. It can prevent microbial growth [6], enhance wound healing [7-9], and inhibit adhesion and activation of platelets [10-13] and proliferation of smooth muscle cells (SMCs) [14]. The perfect

$\dagger$ Contributed equally to this work

*Corresponding author (email: wangshufang@nankai.edu.cn) thromboresistance of the natural endothelium has been partly attributed to the low but continuous production of NO in this layer $[15,16]$. Endothelial cells (ECs) that line the inner walls of healthy blood vessels produce $\mathrm{NO}$ at an estimated flux of $0.5 \times 10^{-10}-4.0 \times 10^{-10} \mathrm{~mol} \mathrm{~cm}{ }^{-2} \mathrm{~min}^{-1}$ [17], which plays an important regulatory role on the physiology and function of the cardiovascular system [18]. This sustained NO-releasing can reduce the occurrence of vascular restenosis $[13,19,20]$. Therefore, vascular grafts that could release or generate NO in situ will possibly solve the problem of thrombosis and intimal hyperplasia of small-diameter vascular grafts [21].

In recent years, some researches have been done in the 
field of NO-releasing polymers [5,22-24]. Some NOreleasing materials have been developed by fixing NO donors such as N-diazeniumdiolate or S-nitrosothiol (RSNO) into the polymer by physical mixing or chemical attachment $[25,26]$. These materials showed excellent anti-thrombosis property, but had problems in burst release, limited storage and inevitable cytotoxicity [27].

There are various kinds of RSNO in peripheral blood, such as S-nitrosoalbumin (Alb-SNO) and S-nitrosoglutathione (GSNO) [28,29], and a selenium-containing enzyme glutathione peroxidase has the effect on catalyzing NO generation from RSNO [30,31]. Because of the constant content of RSNO, this endogenous NO donor can be utilized to fulfill good anticoagulant property by long-term in situ NO generation.

To simulate the function of native blood vessel, we proposed to modify the surface of vascular graft with organoselenium catalyst to generate NO stably from endogenous NO donor RSNO. As a facile surface coating technique, layer-by-layer self-assembly has many advantages such as simple procedures and adaptability to various substrates [32], and has increasingly been used to immobilize biomacromolecules onto the vascular prostheses and tissue engineering scaffolds to improve their hemocompatibility or cytocompatibility [33-38].

In this study, organoselenium immobilized polyethyleneimine and sodium alginate were deposited onto the surface of electrospun polycaprolactone matrix via layer-by-layer self-assembly. In vitro NO generation was performed to test the catalyzing capability of the material. The bioactivity of the modified material was evaluated by adhesion and spreading of SMCs and proliferation of ECs. Finally, the antithrombotic properties of the material were observed through platelet adhesion and arteriovenous shunt experiments.

\section{Materials and methods}

\subsection{Materials}

Polycaprolactone (PCL, $M_{\mathrm{w}} 80 \mathrm{kD}$ ) was purchased from Solvay Interox Ltd, UK. Polyethyleneimine (PEI, $M_{\mathrm{w}} 25 \mathrm{kD}$ ) and polydiallyldimethylammonium chloride (PDDA, $M_{\mathrm{w}}$ 100-200 kD) were obtained from Sigma-Aldrich. 2-(Ncyclohexylamino)-ethanesulfonic acid (CHES), sodium alginate $(\mathrm{AlgNa})$ and glutathione $(\mathrm{GSH})$ were purchased from Beijing Dingguo Biotech Co. Ltd. MTT was obtained from Lianxing Biotechnology Inc. DiI was purchased from Invitrogen. Griess Assay Kit was purchased from Beyotime Institute of Biotechnology.

\subsection{Fibrous scaffold preparation}

PCL fibrous scaffolds (tube for arteriovenous shunt experiment and film for all other parts) were prepared by electro- spinning. PCL was dissolved in chloroform/methanol $(5: 1$ $\mathrm{v} / \mathrm{v})$ to a concentration of $12 \%(\mathrm{w} / \mathrm{v})$. Electrospinning was carried out at a flow rate of $2 \mathrm{~mL} \mathrm{~h}^{-1}$ and a voltage of $18 \mathrm{kV}$. For film matrix, fibers were collected on an aluminum foil on a rotating mandrel with a collecting distance of $20 \mathrm{~cm}$. For tubular matrix, fibers were deposited on a rotating stainless steel mandrel with a diameter of $2.0 \mathrm{~mm}$ at a distance of $18 \mathrm{~cm}$.

\subsection{Loading of organoselenium catalyst via layer-by- layer self-assembly}

3,3'-diselenodipropionic acid (SeDPA) and organoselenium immobilized polyethyleneimine (SePEI) were synthesized according to reported method [39].

Polyelectrolytes were dissolved to make $1 \mathrm{mg} \mathrm{mL}^{-1}$ solutions: PDDA in CHES while SePEI and AlgNa in distilled water. Self-assembly was proceeded by immersing the film alternately into polycation (SePEI or PDDA) and polyanion (AlgNa) solutions for $10 \mathrm{~min}$ and washing by distilled water after each step. A PDDA/AlgNa bilayer was loaded as a precursor layer, and then SePEI and $\mathrm{AlgNa}$ were deposited until a certain number of bilayers $\left((\mathrm{SePEI} / \mathrm{AlgNa})_{n}\right)$ were reached. Films prepared using PEI and AlgNa ((PEI/ $\mathrm{A} \operatorname{lgNa})_{n}$ ) were used as control. For tubular matrix, the polyelectrolyte solutions flowed through the tube at a very slow rate controlled by constant-flow pumps.

\subsection{Characterization of PCL scaffold and polyelectro- lytes loaded film}

\subsubsection{SEM observation}

The micromorphology of PCL scaffold and polyelectrolytes loaded films was observed by scanning electron microscope (SEM; QUANTA 200; FEI, USA) with an acceleration voltage of $15 \mathrm{kV}$. Samples were gold-coated before SEM.

\subsubsection{Contact angle measurement}

To measure water contact angle, self-assembly was done on a film coated on a glass slide. Contact angle was measured on a HARKE SPCA instrument (Beijing, China) after each layer was deposited and dried. The values were determined by averaging the measurements at five different positions $(n=5)$.

\subsubsection{Selenium content measurement}

Selenium (Se) content on the film was measured by an atomic absorption spectrophotometer (TAS-990, Beijing Purkinje General Instrument Co. Ltd., Beijing, China) after each SePEI/AlgNa bilayer was deposited.

\subsubsection{Stability test of loaded catalyst}

PCL-(SePEI/AlgNa $)_{5}$ film was immersed into PBS and kept at $37^{\circ} \mathrm{C}$ under static condition. $5 \mathrm{~mL}$ sample PBS was re- 
moved every five days, and $5 \mathrm{~mL}$ fresh PBS was added. The content of shed Se in sample PBS was scanned by atomic absorption spectrophotometer.

\subsection{In vitro catalytic NO generation determination}

GSNO was synthesized according to literature [40] and slightly modified. $300 \mu \mathrm{L}$ PBS containing $200 \mu \mathrm{mol} \mathrm{L} \mathrm{L}^{-1}$ GSNO, $200 \mu \mathrm{mol} \mathrm{L}{ }^{-1} \mathrm{GSH}$ and $2 \mathrm{nmol} \mathrm{L}^{-1}$ EDTA was added onto each film (PCL-(SePEI/AlgNa) $)_{5}$, PCL-(SePEI/ $\mathrm{AlgNa})_{10}$ and PCL-(SePEI/AlgNa $)_{15}$ ) in 48-well plates and the plates were kept in dark at $37^{\circ} \mathrm{C}$. At certain time points $(2,4,6$ and $8 \mathrm{~h})$, accumulative NO generation was measured using Griess Assay Kit. Tissue culture polystyrene plate (TCPS) and PCL-(PEI/AlgNa $)_{n}$ films were used as controls.

\subsection{Fibroblast proliferation}

For cellular experiments (section 1.6-1.9), sample films were cut into circular pieces, put into 48-well plates and sterilized under ultraviolet radiation overnight.

$3 \mathrm{~T} 3$ fibroblasts were seeded at a concentration of $1 \times 10^{4}$ per film in 48 -well plates and incubated at $37^{\circ} \mathrm{C}$. The medium was refreshed every two days and the proliferation of cells was tested at day 1, 3 and 5 by MTT assay.

\subsection{SMCs adhesion}

Human SMCs were dyed by DiI before seeding onto each film in 48-well plates at a density of $2 \times 10^{4}$ per well with $200 \mu \mathrm{mol} \mathrm{L}{ }^{-1} \mathrm{GSNO}$ and $200 \mu \mathrm{mol} \mathrm{L}{ }^{-1} \mathrm{GSH}$ for $2 \mathrm{~h}$ at $37^{\circ} \mathrm{C}$. The adhered cells were fixed in $4 \%$ paraformaldehyde for $10 \mathrm{~min}$ and observed with inverted fluorescence microscope.

\subsection{SMCs spreading}

$5 \times 10^{4}$ SMCs with $200 \mu \mathrm{mol} \mathrm{L}{ }^{-1}$ GSNO and $200 \mu \mathrm{mol} \mathrm{L}{ }^{-1}$ GSH were seeded onto each film and cultured for $4 \mathrm{~h}$ at $37^{\circ} \mathrm{C}$. The cells were then stained with FITC-labeled phalloidin and observed under confocal laser scanning microscopy (CLSM; TCS SP5; Leica, Germany), or fixed in $2.5 \%$ glutaraldehyde overnight and dehydrated in gradient ethanol for SEM observation.

\subsection{ECs proliferation}

$2 \times 10^{3}$ human umbilical vein endothelial cells were seeded onto each film in 48-well plates with $200 \mu \mathrm{mol} \mathrm{L}{ }^{-1}$ GSNO

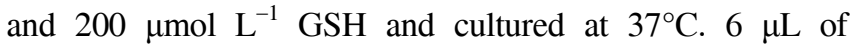
$20 \mathrm{mmol} \mathrm{L}^{-1}$ GSNO and $3 \mu \mathrm{L}$ of $100 \mathrm{mmol} \mathrm{L}^{-1} \mathrm{GSH}$ were added every $12 \mathrm{~h}$ and the medium was replaced every $24 \mathrm{~h}$. After 1, 3, 5 days, the proliferation of cells was determined by MTT method.

\subsection{In vitro platelet adhesion}

Human platelet rich plasma was obtained from Tianjin Blood Center. Platelet suspension $\left(10^{7} \mathrm{~mL}^{-1}\right)$ was added onto each film with $200 \mu \mathrm{mol} \mathrm{L}{ }^{-1}$ GSNO and $200 \mu \mathrm{mol} \mathrm{L}^{-1}$ GSH in 48 -well plate and incubated at $37^{\circ} \mathrm{C}$ under static condition for $1 \mathrm{~h}$. Then the films were washed twice with PBS to remove unattached platelets, and fixed and dehydrated for SEM observation.

\subsection{Arteriovenous shunt (AV-shunt) experiment}

Arteriovenous shunt (AV-shunt) experiment was performed using tubular grafts on male Sprague Dawley rats to investigate the blood compatibility of the modified grafts. All animal procedures were approved by the Animal Care Committee in Nankai University. The connected grafts of PCL and PCL-(SePEI/AlgNa $)_{5}$ were pretreated by heparin for $30 \mathrm{~min}$. Rats were anesthetized by abdominal injection of $10 \%$ chloral hydrate $\left(3 \mathrm{~mL} \mathrm{~kg}^{-1}\right)$. Heparin (100 units kg ${ }^{-1}$ ) was administered as an anti-coagulant agent. After incision of abdominal skin and isolation of abdominal aorta and inferior vein, the series of grafts was connected into the circulation using indwelling needles. After circulation of $1 \mathrm{~h}$, the circuit was perfused with physiological saline and fixed with $4 \%$ paraformaldehyde for $4 \mathrm{~h}$ for stereomicroscope observation or fixed in $2.5 \%$ glutaraldehyde overnight and dehydrated through gradient ethanol for SEM observation.

\subsection{Statistical analysis}

All experiments were performed using six samples $(n=6)$ and quantitative results were presented as mean \pm standard deviation (SD) unless otherwise specified. Statistical significance analysis was determined using TTEST. Significance was established by a value of $P<0.05$.

\section{Results and discussion}

\subsection{Characterizations of prepared materials}

Uniformly distributed smooth PCL electrospun fibers with diameters in the range of 100-400 nm (Figure 1G) were obtained under the condition in this study. After loading of polyelectrolytes, there were slight changes in the morphology of the fibers. Fibers of (SePEI/AlgNa $)_{n}$ films were clearer than those of $(\mathrm{PEI} / \mathrm{AlgNa})_{n}$ films, while the latter exhibited some extent of adhesion and conglutination. This could be possibly explained by the fact that the derivation of PEI with SeDPA reduced the viscosity of its solution and thus resulted in a clearer micromorphology.

Water contact angle of the prepared film was measured 


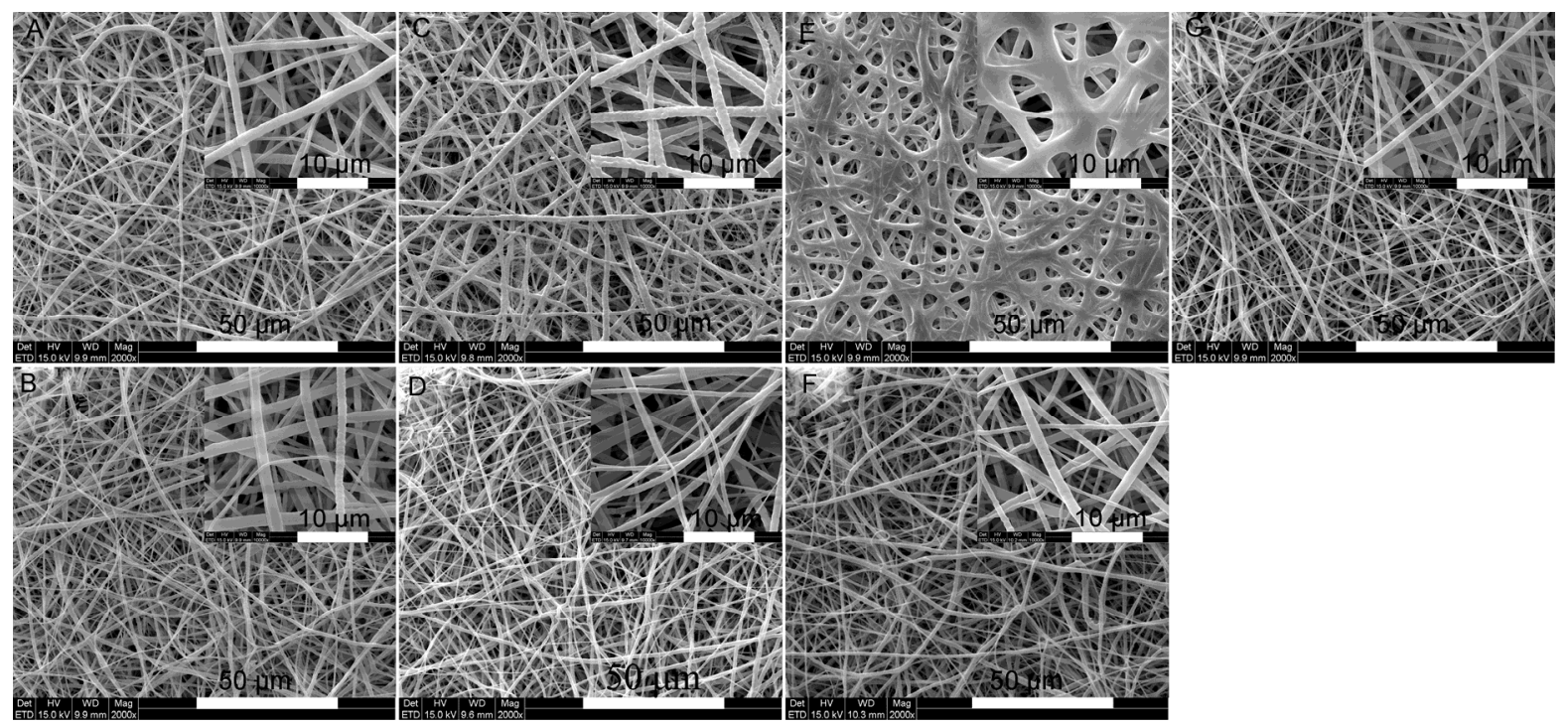

Figure 1 Micromorphology of prepared films. A, (PEI/AlgNa) $)_{5}$. B, (SePEI/AlgNa) $)_{5}$ C, $(\mathrm{PEI} / \mathrm{AlgNa})_{10}$. D, $(\mathrm{SePEI} / \mathrm{AlgNa})_{10}$. E, $(\mathrm{PEI} / \mathrm{AlgNa})_{15} . \mathrm{F}$, $(\mathrm{SePEI} / \mathrm{AlgNa})_{15}$. G, PCL. (Scale bar $=50 \mu \mathrm{m}$ in low magnification images and $10 \mu \mathrm{m}$ in high magnification images).

to determine the hydrophilicity/hydrophobicity and make an analysis of the assembly process. The contact angle was $103^{\circ} \pm 5^{\circ}$ when polycation SePEI was the outermost layer and $93^{\circ} \pm 3^{\circ}$ for polyanion $\mathrm{AlgNa}$ (Figure 2). The zigzag curve of contact angle proved the alternate adsorption of the polyelectrolytes.

To have a quantitative analysis of the assembly, atomic absorption spectrometry was used to get the exact content of Se loaded on the film (Figure 3). The content of Se increased rapidly for the first three bilayers but relatively slowly for the next five bilayers, and reached a maximum amount of $1.53 \times 10^{3} \mu \mathrm{g} \mathrm{L}^{-1} \mathrm{~cm}^{-2}$ on each piece of film (diameter $1 \mathrm{~cm}$ ) after the eighth bilayer. The content decreased at the ninth bilayers a bit, probably due to self-healing of electrostatic assembly film, which is that the surface charge density may reach a constant after several depositions [41].

Retention rate of Se element on the film was determined to evaluate the stability of loaded Se catalyst. Retention rate was calculated as the ratio of Se content on the film after certain days immersed in PBS to the original content on the film. The result showed an acceptable stability of loaded catalyst under static condition, with a Se retention rate of $80 \%$ after immersing in PBS for up to 35 days.

\subsection{Catalytic NO generation}

Results of in vitro catalytic NO generation were shown in Figure 4. With EDTA in the system, interfering catalytic decomposition of GSNO by transient metal ions such as $\mathrm{Cu}$ (II) could be eliminated. As illustrated in Figure 4A, B and $\mathrm{C}$, catalyst-loaded materials ((SePEI/AlgNa) $)_{n}$ ) could obviously accelerate the release of NO from its donor GSNO. Therefore, materials constructed by the method in this study are expected to fulfill in situ NO generation when contact-

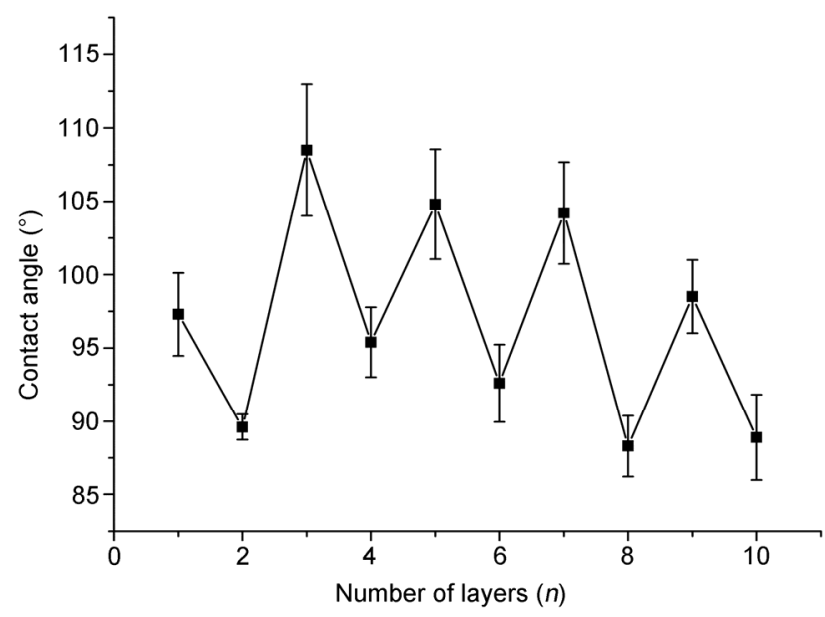

Figure 2 Water contact angles of (SePEI/AlgNa $)_{n}$ film with different numbers of layers of polyelectrolytes. Odd numbers signify SePEI as the outermost layer and even numbers signify $\mathrm{AlgNa}$.

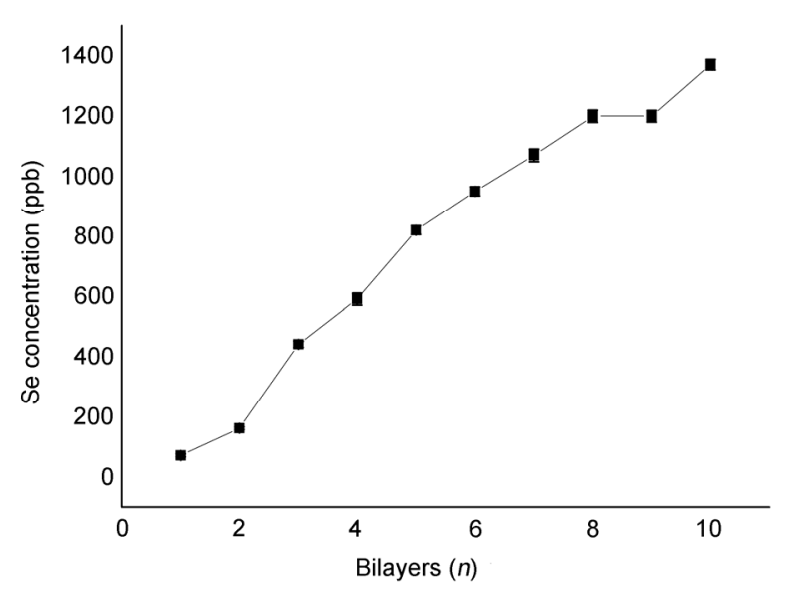

Figure 3 Se content on (SePEI/AlgNa $)_{10}$ film after each loaded bilayer. 

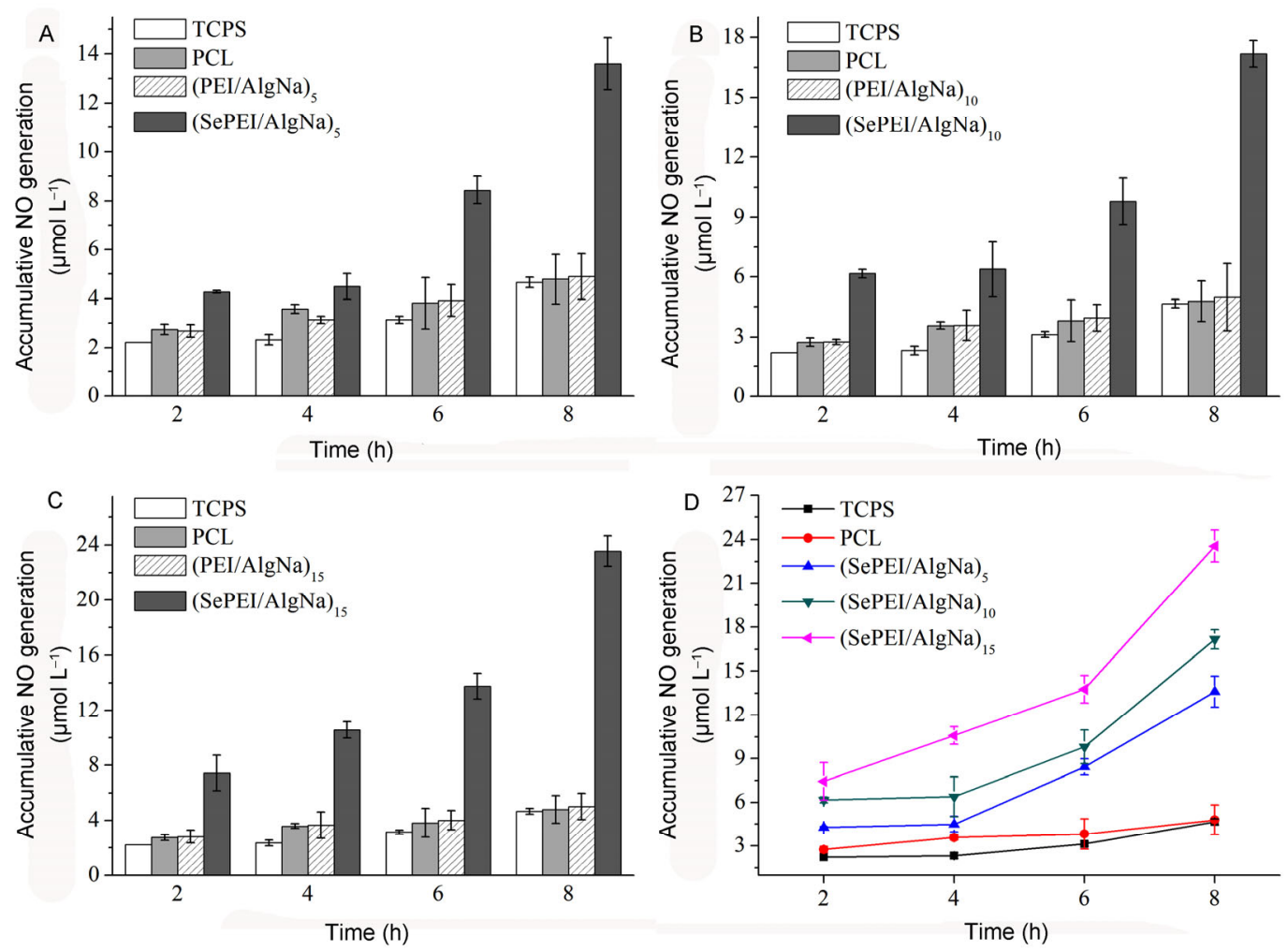

Figure 4 (color online) In vitro catalytic NO generation by films with different numbers of loaded bilayers. A, (SePEI/AlgNa) ${ }_{5}$ B, (SePEI/AlgNa) $)_{10}$ C, $(\mathrm{SePEI} / \mathrm{AlgNa})_{15}$. D, collected results of $(\mathrm{SePEI} / \mathrm{AlgNa})_{n}$. (Data expressed as mean $\left.\pm \mathrm{SD}, n=6\right)$.

ing peripheral blood, which contains a certain amount of NO donors.

By comparing results of different groups (Figure 4D), it could be seen that the catalyzing ability of the material was increased with a rise in the number of bilayers loaded. The amount of Se catalyst on the film was proportional to the number of loaded bilayers, and therefore this approach would enable a controllable catalyzing capability and NO generation.

\subsection{Cellular behavior evaluation}

Proliferation of 3T3 fibroblast was tested using MTT assay to evaluate the cytocompatibility of the materials. Figure 5 shows that the cell number was higher on PCL$(\mathrm{SePEI} / \mathrm{AlgNa})_{5}$ film than that on PCL-(PEI/AlgNa $)_{5}$ or control groups (PCL and TCPS). This result suggested that the polycation SePEI and polyanion AlgNa possess satisfactory cellular compatibility, and their contribution to the increased hydrophilicity of the film surface might be the reason of a higher cell amount.

The effects of catalytically generated NO on adhesion and spreading of SMCs were evaluated. The adhesion of SMCs was reduced on PCL-(SePEI/AlgNa) film, demonstrated by a lower cell number (Figure 6). Meanwhile, observation of cell morphology under SEM and CLSM discovered that the spreading of SMCs was inhibited on catalyst-loaded film (Figure 7). SMCs exhibited full spreading

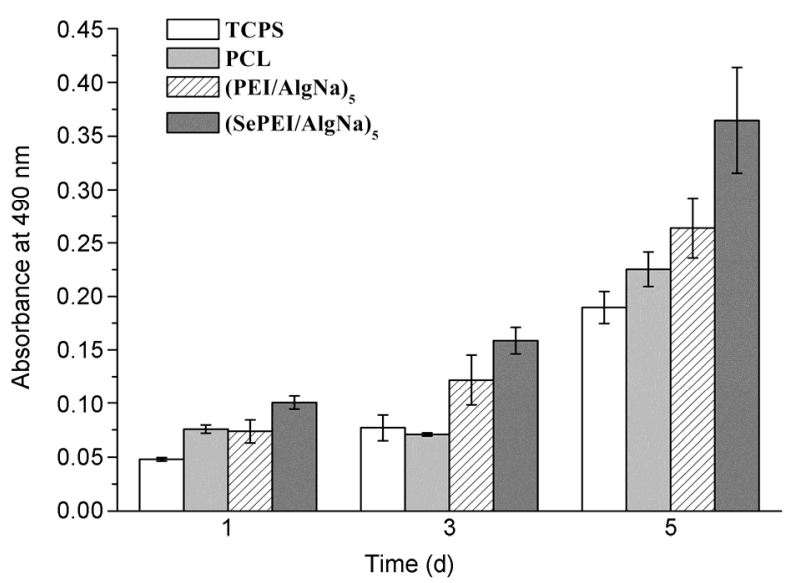

Figure 5 Cytocompatibility of (SePEI/AlgNa) $)_{5}$ film. (Data expressed as mean $\pm \mathrm{SD}, n=6)$.

on PCL or PCL-(PEI/AlgNa) $)_{5}$ film, but shrunk shape on PCL-(SePEI/AlgNa $)_{5}$ film. Since SMCs have been indicated as the major participant cells in intimal hyperplasia and restenosis, and NO is reported to suppress the adhesion and proliferation of SMCs [20], materials constructed in this study would be effective in reducing intimal hyperplasia by catalyzing long-term in situ NO generation.

ECs proliferation on the films was monitored to observe the effect of catalyst-loaded film on ECs growth. It could be seen from the result (Figure 8 ) that the cell number on 

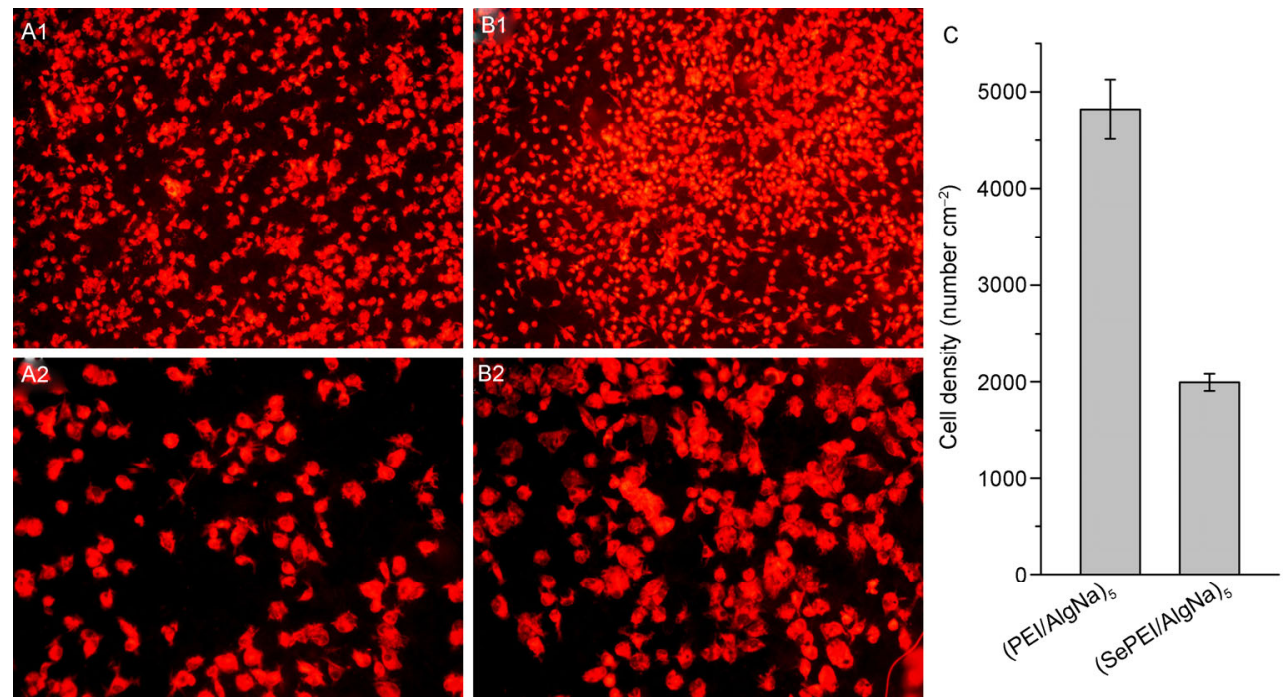

Figure 6 (color online) SMCs adhesion on $(\mathrm{SePEI} / \mathrm{AlgNa})_{5}$ film. $\mathrm{A}_{1},(\mathrm{SePEI} / \mathrm{AlgNa})_{5}, 10 \times . \mathrm{A}_{2},(\mathrm{SePEI} / \mathrm{AlgNa})_{5}, 20 \times . \mathrm{B}_{1},(\mathrm{PEI} / \mathrm{AlgNa})_{5}, 10 \times . \mathrm{B}_{2}$, $(\mathrm{PEI} / \mathrm{AlgNa})_{5}, 20 \times . \mathrm{C}$, cell density.

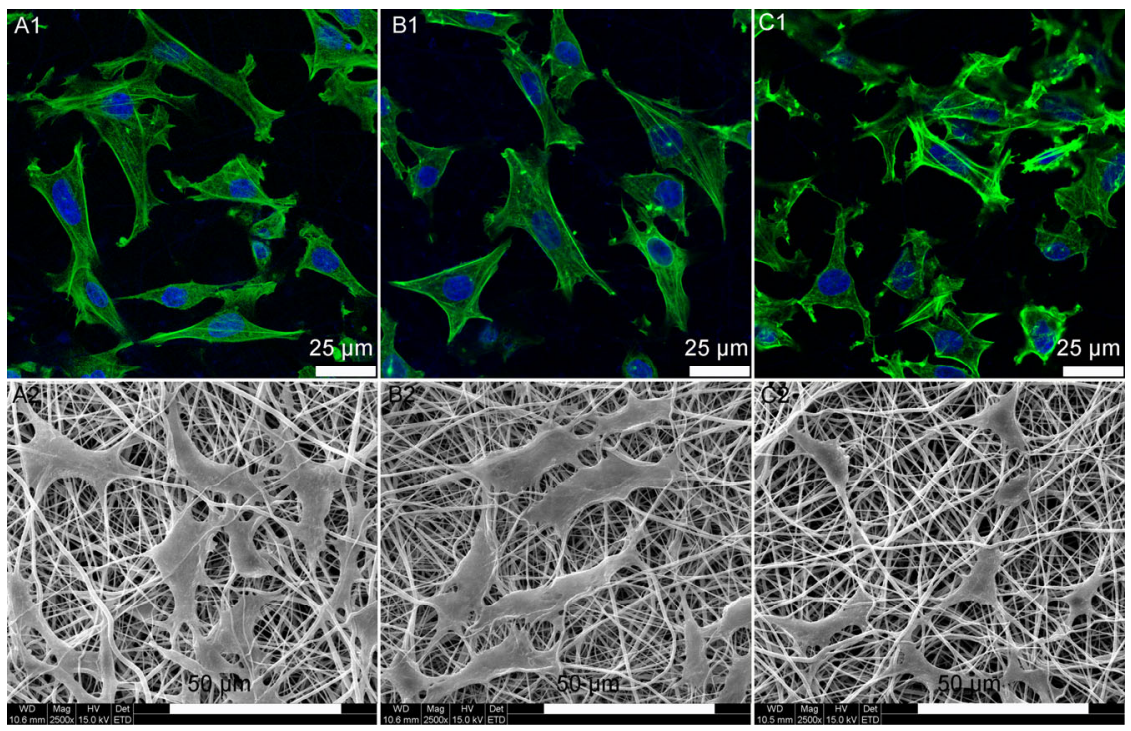

Figure 7 (color online) SMCs spreading on (SePEI/AlgNa) $)_{5}$ film. A, PCL. B, (PEI/AlgNa) $)_{5}$ C, (SePEI/AlgNa) $)_{5}$ 1: CLSM images; 2: SEM photos. (Scale bar $=25 \mu \mathrm{m}$ in CLSM images and $50 \mu \mathrm{m}$ in SEM images).

PCL-(SePEI/AlgNa $)_{5}$ film was greater compared to those on PCL and PCL-(PEI/AlgNa $)_{5}$ films. ECs play significant functions in blood vessels as preventing thrombosis and modulating proliferation of SMCs, and thus rapid endothelialization has become one concern in vascular graft construction. NO was reported to stimulate the proliferation of ECs [20], therefore, surface modification method proposed in this study might be helpful in promoting endothelialization of vascular grafts.

\subsection{Evaluation of antithrombotic properties}

The antithrombotic properties of NO generating grafts constructed in this study were characterized by in vitro platelet adhesion and AV-shunt experiments. SEM graphs in Figure 9

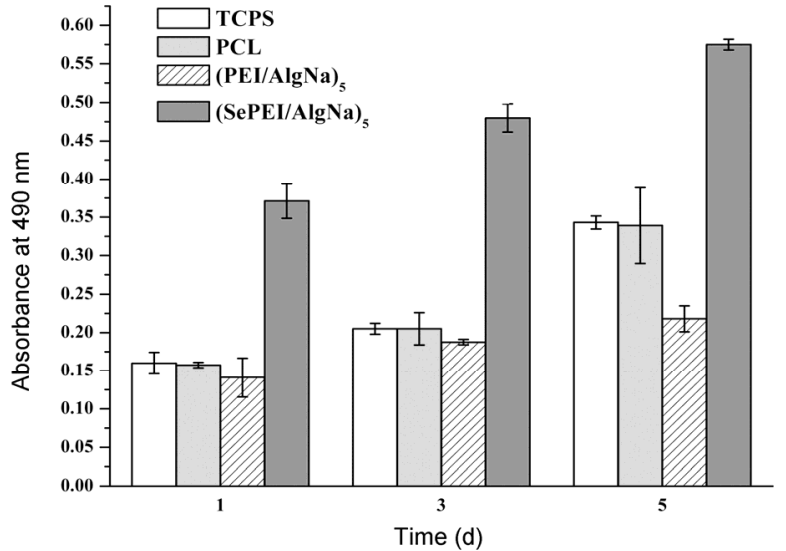

Figure 8 Proliferation of ECs on $(\mathrm{SePEI} / \mathrm{AlgNa})_{5}$ film. (Data expressed as mean $\pm \mathrm{SD}, n=6$ ). 
show that the quantity of adhered platelets was less on surfaces of PCL-(SePEI/AlgNa) $)_{5}$ film compared to those on the controls.

To evaluate the property of the modified graft to prevent acute thrombosis, tubular PCL-(SePEI/AlgNa) $)_{5}$ graft was exposed to blood flow for $1 \mathrm{~h}$ in $\mathrm{AV}$-shunt experiment. Stereomicroscopy showed that the luminal surface of the grafts was clean and smooth without visible thrombi (Figure $10 \mathrm{~B}_{1}$ ). SEM images showed that abundant platelets adhered on PCL grafts (Figure $10 \mathrm{~A}_{2}$ ) whereas few platelets were observed on PCL-(SePEI/AlgNa) $)_{5}$ graft (Figure $10 \mathrm{~B}_{2}$ ), suggesting an inhibitory property of the NO generating PCL-(SePEI/AlgNa) $)_{5}$ surface on platelet adhesion. As adhesion and activation of platelets is a critical and starting step in thrombus formation, the catalyst-loaded grafts might reduce the risk of acute thrombosis and possess a better hemocompatibility.

\section{Conclusion}

In this study, we proposed a novel method of improving the hemocompatibility and bioactivity of artificial blood vessel by loading organoselenium catalyst onto the inner surface of electrospun tubular graft. Catalyst-loaded material showed significant in situ NO generation and the catalyzing capability could be adjusted through the number of polyelectrolyte layers loaded. Modified films possessed good cellular compatibility, and could inhibit adhesion and spreading of SMCs and promote proliferation of ECs. Satisfactory antithrombotic properties of these NO generating materials were proven by reduced platelet adhesion and prevention of acute thrombosis. This study provides a potential approach of constructing vascular grafts with improved antithrombotic property and desirable biological functions.

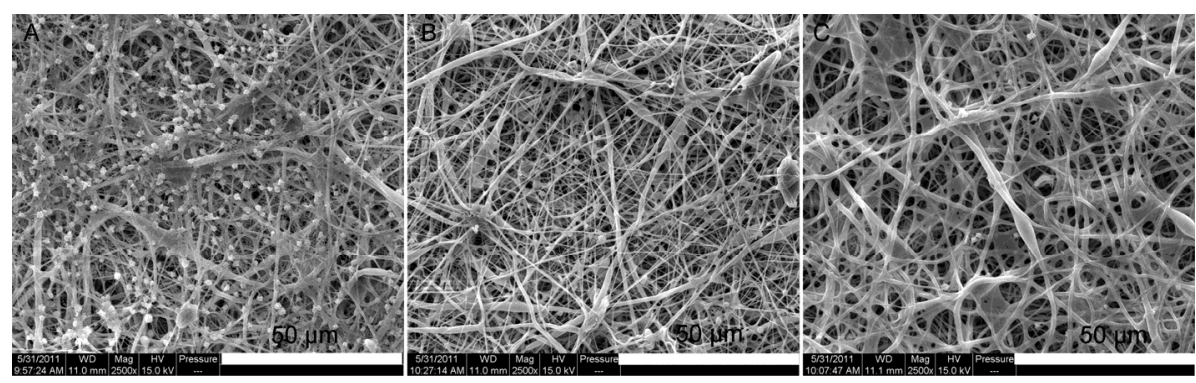

Figure 9 Platelet adhesion on $(\mathrm{SePEI} / \mathrm{AlgNa})_{5}$ film. A, PCL. B, $(\mathrm{PEI} / \mathrm{AlgNa})_{5} . \mathrm{C},(\mathrm{SePEI} / \mathrm{AlgNa})_{5} .(\mathrm{Scale}$ bar $=50 \mu \mathrm{m})$.

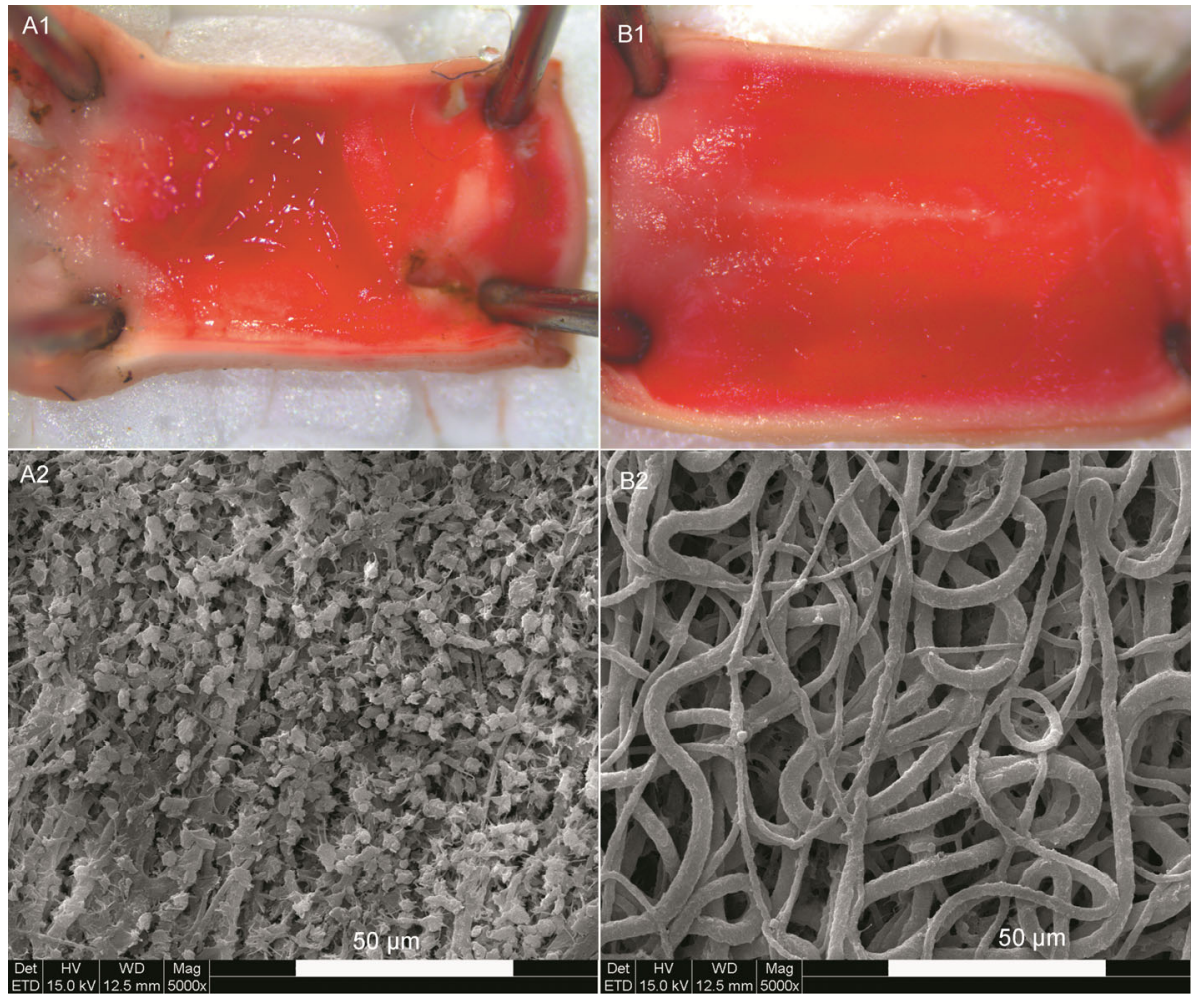

Figure 10 (color online) Hemocompatibility of (SePEI/AlgNa) $)_{5}$ graft. A, PCL. B, (SePEI/AlgNa) $)_{5}$ 1: stereomicroscope images; 2: SEM photos. (Scale bar $=50 \mu \mathrm{m}$ in SEM images). 
This work was supported by the National Basic Research Program of China (2012CB725204), National Natural Science Foundation of China (51073081, 31170030 and J1103503), Program for Changjiang Scholars and Innovative Research Team in University (IRT13023), and the Natural Science Foundation of Tianjin, China (13JCYBJC24900 and 13JCZDJC27800).

1 Cleary MA, Geiger E, Grady C, Best C, Naito Y, Breuer C. Vascular tissue engineering: the next generation. Trends Mol Med, 2012, 18: 394-404

2 Krejcy K, Schmetterer L, Kastner J, Nieszpaur-Los M, Monitzer B, Schutz W, Eichler HG, Kyrle PA. Role of nitric oxide in hemostatic system activation in vivo in humans. Arterioscler Thromb Vasc Biol, 1995, 15: 2063-2067

3 Salvemini D, Masini E, Anggard E, Mannaioni PF, Vane J. Synthesis of a nitric oxide-like factor from L-arginine by rat serosal mast cells: Stimulation of guanylate cyclase and inhibition of platelet aggregation. Biochem Biophys Res Commun, 1990, 169: 596-601

4 Samama CM, Diaby M, Fellahi JL, Mdhafar A, Eyraud D, Arock M, Guillosson JJ, Coriat P, Rouby JJ. Inhibition of platelet aggregation by inhaled nitric oxide in patients with acute respiratory distress syndrome. Anesthesiology, 1995, 83: 56-65

5 Brisbois EJ, Handa H, Major TC, Bartlett RH, Meyerhoff ME. Long-term nitric oxide release and elevated temperature stability with S-nitroso-N-acetylpenicillamine (SNAP)-doped Elast-eon E2As polymer. Biomaterials, 2013, 34: 6957-6966

6 Mancinelli RL, McKay CP. Effects of nitric oxide and nitrogen dioxide on bacterial growth. Appl Environ Microbiol, 1983, 46: 198-202

7 Rizk M, Witte MB, Barbul A. Nitric oxide and wound healing. World J Surg, 2004, 28: 301-306

8 Witte MB, Barbul A. Role of nitric oxide in wound repair. Am J Surg, 2002, 183: 406-412

9 Broughton G, Janis JE, Attinger CE. The basic science of wound healing. Plast Reconstr Surg, 2006, 117: 12s-34s

10 Diodati JG, Quyyumi AA, Hussain N, Keefer LK. Complexes of nitric oxide with nucleophiles as agents for the controlled biological release of nitric oxide: antiplatelet effect. Thromb Haemost, 1993, 70: 654-658

11 Ignarro LJ, Buga GM, Wood KS, Byrns RE, Chaudhuri G. Endothelium-derived relaxing factor produced and released from artery and vein is nitric oxide. Proc Natl Acad Sci USA, 1987, 84: 9265-9269

12 Radomski MW, Palmer RM, Moncada S. An L-arginine/nitric oxide pathway present in human platelets regulates aggregation. Proc Natl Acad Sci USA, 1990, 87: 5193-5197

13 Feldman PL, Griffith OW, Stuehr DJ. The surprising life of NITRIC OXIDE. Chem Eng News, 1993, 71: 26-38

14 Sarkar R, Webb RC. Does nitric oxide regulate smooth muscle cell proliferation? A critical appraisal. J Vasc Res, 1998, 35: 135-142

15 Radomski MW, Vallance P, Whitley G, Foxwell N, Moncada S. Platelet adhesion to human vascular endothelium is modulated by constitutive and cytokine induced nitric oxide. Cardiovasc Res, 1993, 27: $1380-1382$

16 Michiels C. Endothelial cell functions. J Cell Physiol, 2003, 196: 430-443

17 Vaughn MW, Kuo L, Liao JC. Estimation of nitric oxide production and reaction rates in tissue by use of a mathematical model. Am J Physiol, 1998, 274: H2163-H2176

18 Miller MR, Megson IL. Recent developments in nitric oxide donor drugs. Br J Pharmacol, 2007, 151: 305-321

19 Moncada S, Palmer RM, Higgs EA. Nitric oxide: physiology, pathophysiology, and pharmacology. Pharmacol Rev, 1991, 43: 109-142

20 Bredt DS, Snyder SH. Biological roles of nitric oxide. Sci Am, 1992, 266: $68-77$
21 Conklin BS, Richter ER, Kreutziger KL, Zhong DS, Chen C. Development and evaluation of a novel decellularized vascular xenograft. Med Eng Phys, 2002, 24: 173-183

22 Cai WY, Wu JF, Xi CW, Meyerhoff ME. Diazeniumdiolate-doped poly(lactic-co-glycolic acid)-based nitric oxide releasing films as antibiofilm coatings. Biomaterials, 2012, 33: 7933-7944

23 Cai WY, Wu JF, Xi CW, Ashe AJ, Meyerhoff ME. Carboxyl-ebselen-based layer-by-layer films as potential antithrombotic and antimicrobial coatings. Biomaterials, 2011, 32: 7774-7784

24 Hofler L, Meyerhoff ME. Modeling the effect of oxygen on the amperometric response of immobilized organoselenium-based S-nitrosothiol sensors. Anal Chem, 2011, 83: 619-624

25 Reynolds MM, Frost MC, Meyerhoff ME. Nitric oxide-releasing hydrophobic polymers: Preparation, characterization, and potential biomedical applications. Free Radical Biol Med, 2004, 37: 926-936

26 Zhang L, Wang K, Zhao Q, Zheng WT, Wang ZH, Wang SF, Kong DL. Core-shell fibrous vascular grafts with the nitric oxide releasing property. Sci China Chem, 2010, 53: 528-534

27 Seabra AB, da Silva R, de Souza GFP, de Oliveira MG. Antithrombogenic polynitrosated polyester/poly(methyl methacrylate) blend for the coating of blood-contacting surfaces. Artif Organs, 2008, 32: 262-267

28 Giustarini D, Milzani A, Colombo R, Dalle-Donne I, Rossi R. Nitric oxide and S-nitrosothiols in human blood. Clin Chim Acta, 2003, 330: $85-98$

29 Kelm M. Nitric oxide metabolism and breakdown. Biochimica Et Biophysica Acta-Bioenergetics, 1999, 1411: 273-289

30 Mugesh G, Singh HB. Synthetic organoselenium compounds as antioxidants: glutathione peroxidase activity. Chem Soc Rev, 2000, 29: 347-357

31 Oh BK, Meyerhoff ME. Spontaneous catalytic generation of nitric oxide from S-nitrosothiols at the surface of polymer films doped with lipophilic copper(II) complex. J Am Chem Soc, 2003, 125: 9552-9553

32 Lin QK, Van JJ, Qiu FY, Song XX, Fu GS, Ji JA. Heparin/collagen multilayer as a thromboresistant and endothelial favorable coating for intravascular stent. J Biomed Mater Res Part A, 2011, 96A: 132-141

33 Picart C. Polyelectrolyte multilayer films: From physico-chemical properties to the control of cellular processes. Curr Med Chem, 2008, 15: 685-697

34 Tang ZY, Wang Y, Podsiadlo P, Kotov NA. Biomedical applications of layer-by-layer assembly: From biomimetics to tissue engineering. Adv Mater, 2006, 18: 3203-3224

35 Thierry B, Winnik FM, Merhi Y, Silver J, Tabrizian M. Bioactive coatings of endovascular stents based on polyelectrolyte multilayers. Biomacromolecules, 2003, 4: 1564-1571

36 Meng S, Liu ZJ, Shen L, Guo Z, Chou LSL, Zhong W, Du QG, Ge J. The effect of a layer-by-layer chitosan-heparin coating on the endothelialization and coagulation properties of a coronary stent system. Biomaterials, 2009, 30: 2276-2283

37 Chen JL, Li QL, Chen JY, Chen C, Huang N. Improving blood-compatibility of titanium by coating collagen-heparin multilayers. Appl Surf Sci, 2009, 255: 6894-6900

38 Yang J, Welby JL, Meyerhoff ME. Generic nitric oxide (NO) generating surface by immobilizing organoselenium species via Layer-by-Layer assembly. Langmuir, 2008, 24: 10265-10272

39 Koch T, Suenson E, Henriksen U, Buchardt O. The oxidative cleavability of protein cross-linking reagents containing organoselenium bridges. Bioconj Chem, 1990, 1: 296-304

40 Heikal L, Martin GP, Dailey LA. Characterisation of the decomposition behaviour of S-nitrosoglutathione and a new class of analogues: S-Nitrosophytochelatins. Nitric Oxide, 2009, 20: 157-165

41 Zheludkevich ML, Shchukin DG, Yasakau KA, Mohwald H, Ferreira MGS. Anticorrosion coatings with self-healing effect based on nanocontainers impregnated with corrosion inhibitor. Chem Mater, 2007, 19: 402-411

Open Access This article is distributed under the terms of the Creative Commons Attribution License which permits any use, distribution, and reproduction in any medium, provided the original author(s) and source are credited. 